\title{
Molecular-level realignment in donor-acceptor bilayer blends on metals
}

P. Borghetti, ${ }^{a, b^{*}}$ D. G. de Oteyza, ${ }^{a, c, d}$ C. Rogero, ${ }^{c}$ E. Goiri, ${ }^{a}$ A. Verdini, ${ }^{e}$ A. Cossaro, ${ }^{e}$ L. Floreano, ${ }^{e}$ J. E. Ortega ${ }^{a, c, f}$

${ }^{a}$ Donostia International Physics Center, Paseo Manuel Lardizabal 4, 20018 San Sebastián, Spain ${ }^{\mathrm{b}}$ Institut des Nanosciences de Paris, 4 place Jussieu, 75005 Paris, France

${ }^{\mathrm{c}}$ Centro de Física de Materiales (CSIC-UPV/EHU) - MaterialsPhysics Center (MPC), Paseo Manuel Lardizabal 5, 20018 San Sebastián, Spain

${ }^{\mathrm{d}}$ Ikerbasque, Basque Foundation for Science, 48011 Bilbao, Spain

${ }^{\mathrm{e}}$ CNR-IOM, Laboratorio TASC, Basovizza SS-14 Km. 163.5, 34149 Trieste, Italy.

${ }_{\mathrm{f}}^{\mathrm{f}}$ Departamento de Física Aplicada I, Universidad del Pais Vasco, Pza. Oñate 2, 20018 San Sebastián, Spain.

* borghetti@insp.jussieu.fr, Tel.: +33 (0)144274650 


\begin{abstract}
Molecular bilayers including donor-acceptor blends at the interface to metal substrates show a tunable energy level alignment at both metal-organic and organic-organic interfaces. To ensure sharp hetero-molecular interfaces, contact-layers are formed by stable blends of pentacene (PEN) and perfluorinated copper phthalocyanine $\left(\mathrm{F}_{16} \mathrm{CuPc}\right)$ on $\mathrm{Au}(111)$ and $\mathrm{Ag}(111)$ and of perfluoropentacene (PFP) and copper phthalocyanine $(\mathrm{CuPc})$ on $\mathrm{Ag}(111)$. Core-level and valence band photoemission reveal that upon capping with pure $\mathrm{F}_{16} \mathrm{CuPc}, \mathrm{CuPc}$, and $\mathrm{PEN}$, the electronic states of both contact and capping layers "realign" with respect to the monolayer and the multilayer references. The sign of the shift depends clearly on whether the capping layer is donor-like (PEN, $\mathrm{CuPc})$ or acceptor-like $\left(\mathrm{F}_{16} \mathrm{CuPc}\right)$. As revealed by NEXAFS, the shift in electronic levels of the contact layer upon capping leads to a spectral density variation across the Fermi Edge $\left(\mathrm{E}_{\mathrm{F}}\right)$, i.e., it induces molecule/metal charge transfer.
\end{abstract}




\section{Introduction}

The development of organic hetero-junction devices, such as photovoltaic cells and organic light emitting diodes, demands the ability of tailoring physical/chemical properties at both metal-organic and organic-organic interfaces. In the path towards high-efficiency organic devices, architectures as well as electronic level offsets at the various interfaces need to be controlled at the molecular scale to favor the exciton dissociation, the charge transport across the organic film, and the charge injection at the electrode. However, while there is a wealth of studies on the structural and electronic properties of metal-organic interfaces ${ }^{1-5}$, very few studies have been dedicated to the investigation of organic hetero-interfaces with well-defined structures, including the simplest bilayer systems.

Functional organic bilayers can be made by alternate sublimation of aromatic donor/acceptor monolayers on metals, as demonstrated for perfluorinated copper phthalocyanine $\left(\mathrm{F}_{16} \mathrm{CuPc}\right)$ on copper phthalocyanine $(\mathrm{CuPc})$ on $\mathrm{Ag}(111)^{6}$ and for metal-phthalocyanine $(\mathrm{MePc}, \mathrm{Me}=\mathrm{Sn}, \mathrm{Cu})$ on perylene tetracarboxylic dianhydride (PTCDA) on $\operatorname{Ag}(111)^{7-11}$. Such systems exhibit heteromolecular interfaces with direct $\pi$-plane stacking, although electronic and optical coupling at the intermolecular contact is found to be weak ${ }^{9}$. Yet, bilayer hetero-structures show relevant electronic properties, such as the hetero-molecular interface dipole, which shifts the energy levels ${ }^{8}$, and influences the electronic properties of the molecule/metal interface ${ }^{11}$. This suggests the possibility of further manipulating bilayer electronic levels by tailoring the donor/acceptor mixture. Here, we aim at finding general trends for energy level alignment at bilayer/metal interfaces, through a systematic variation of the donor-acceptor composition of the organic stack.

One major challenge for donor/acceptor multilayers is achieving both sharp molecular heterointerfaces and layer-by-layer growth. Although there are examples of epitaxial growth of alternate species $^{6,8,10,12}$, mutual molecular intermixing is likely to happen. In reality, a tunable donor/acceptor 
mixing is desirable, e.g., to control energy level alignment and charge transfer at the metal interface $^{13,14,15}$, or to enable the growth of crystal quality bilayers, as is the case for $\mathrm{F}_{16} \mathrm{CuPc}: \mathrm{PEN}$ bilayers sublimated on $\mathrm{Au}(111)^{16}$. In fact, sequential $\mathrm{F}_{16} \mathrm{CuPc}$ on $\mathrm{PEN}$ stacking is not possible since molecular intermixing occurs. In contrast, a 3:1 PEN:F ${ }_{16} \mathrm{CuPc}$ contact monolayer blend prevents further mixing, i.e., allows the subsequent nucleation of a pure $\mathrm{PEN}$ or $\mathrm{F}_{16} \mathrm{CuPc}$ layer on top of the contact one. The geometry of such second molecular layer remains an open question. The scanning tunneling microscopy (STM) results of Wakayama et al. suggest that both the $\mathrm{F}_{16} \mathrm{CuPc}$ and PEN molecules switch from the planar configuration of the first layer to a tilted geometry in the second one $^{16}$. However, due to the nature of STM images, where topography and density of states are convoluted, complementary methods of structure analysis are required to firmly establish the molecular adsorption geometry.

In this work, we explore the electronic states in donor/acceptor bilayer systems in which the contact with the metal is defined by a stable "donor:acceptor" blend. We analyze the case of PEN/PEN:F $F_{16} \mathrm{CuPc} / \mathrm{Au}(111)$ and $\mathrm{F}_{16} \mathrm{CuPc} / \mathrm{PEN}: \mathrm{F}_{16} \mathrm{CuPc} / \mathrm{Au}(111)$ bilayers. We extend the study to $\mathrm{Ag}(111)$, where we investigate $\mathrm{F}_{16} \mathrm{CuPc} / \mathrm{PEN}: \mathrm{F}_{16} \mathrm{CuPc}$ and its isomorphic combination with inverted donor-acceptor character $\mathrm{CuPc} / \mathrm{CuPc}: \mathrm{PFP}$. For all systems except for PEN/PEN:F ${ }_{16} \mathrm{CuPc}$, a planar molecular adsorption geometry is demonstrated by polarization-dependent near edge X-ray absorption fine structure (NEXAFS) measurements. X-ray Photoemission Spectroscopy (XPS) measurements allows to identify and resolve distinct electronic levels for each molecular layer in the stack. We observe a rigid electronic structure shift of the binding energies in the contact layer upon bilayer capping, where the sign of the shift depends on the relative donor/acceptor character of the topmost layer. Such rigid shift drives further molecule/metal charge transfer, as deduced from the spectral density change in NEXAFS.

\section{Experimental Methods}


The $\mathrm{Au}(111)$ and $\operatorname{Ag}(111)$ surfaces were prepared by standard sputtering and annealing cycles, their cleanliness was checked by XPS or STM prior to molecular deposition. $\mathrm{CuPc}, \mathrm{F}_{16} \mathrm{CuPc}$, and PEN were purchased from Sigma-Aldrich, and PFP from Kanto Denka Kogyo. Deposition of molecular layers took place from resistively heated Knudsen cells at temperatures around 650 and $470 \mathrm{~K}$ for the phthalocyanines and acenes, respectively, onto single crystal surfaces held at room temperature. The structural and electronic analysis of the molecular bilayers were performed at room temperature in two different Ultra-High Vacuum (UHV) systems: a home-laboratory with STM, UPS and a conventional x-ray source and a synchrotron laboratory for high-resolution photoemission spectroscopy and NEXAFS. In both cases, careful thickness calibration was achieved by use of a quartz crystal microbalance and supported a posteriori by detailed analysis of the relative core-level peak intensities. In the first case, this method is further corroborated by STM images.

The NEXAFS, XPS and valence band (VB) spectra (except for the valence band of the PFP:CuPc systems) have been measured at the ALOISA beamline of the ELETTRA synchrotron in Trieste, Italy. NEXAFS spectra were taken by measuring the partial electron yield with a channeltron detector and they were calibrated a posteriori to the $1 \mathrm{~s} \rightarrow \pi^{*}$ gas phase transitions of $\mathrm{CO}$ and $\mathrm{N}_{2}$ at $\mathrm{h} v=287.4$ and $401.10 \mathrm{eV}$, as described in Ref. ${ }^{17}$. The orientation of the surface with respect to the linear polarization of the synchrotron beam was changed by rotating the sample around the beam axis while keeping a constant grazing angle of $6^{\circ}{ }^{18}$. This scattering geometry allows the change from linear s-polarization (s-pol, i.e. light polarization parallel to the sample surface) to ppolarization (p-pol, i.e. light polarization perpendicular to the sample surface) without any variation of the illuminated area on the sample. In order to compare the intensity of the spectra taken on the different samples, the spectra have been normalized to the intensity at high photon energy (i.e., hv= $305 \mathrm{eV}$ for the $\mathrm{C} \mathrm{K}$-edge and $\mathrm{h} v=420 \mathrm{eV}$ for the $\mathrm{N}$ K-edge). NEXAFS spectra are deconvoluted by performing a nonlinear least-squares fitting of the experimental data, using a set of Gaussian 
functions. An additional integral background was included in order to account for transitions to the continuum of free electron states.

The XPS data were collected by means of a hemispherical electron energy analyzer in normal emission while keeping the sample at grazing incidence $\left(4^{\circ}\right)$. Core level XPS spectra were measured in p-polarization and normal emission, whereas valence band spectra were measured at the magic angle. The excitation energies correspond to $140 \mathrm{eV}$ (valence band) and $530 \mathrm{eV}$ (C 1s) and the overall resolution is 200 and $300 \mathrm{meV}$, respectively. Additionally, valence band spectra of PFP:CuPc systems were measured using the He I $\alpha$ line $(21.2 \mathrm{eV})$ from a non-monochromatic gas discharge lamp and a SPECS Phoibos 100 electron analyzer. In this case, spectra were recorded at $45^{\circ}$ off-normal emission. The He I $\beta$ satellite lines caused by the non-monochromatic photon source were taken into account by reducing the intensity of each point by $2 \%$ of the corresponding intensity at fixed kinetic energy intervals of $-1.87 \mathrm{eV}$, i.e. from the emission associated with the $\mathrm{He}$ I $\alpha$ main line. This data treatment enables to remove the $\mathrm{Ag} d$-band satellites in the vicinity of the photoemission peak associated to PFP HOMO+1. The binding energy of core-level spectra is carefully calibrated taking the substrate core-level energies as absolute references (bulk peaks of $\mathrm{Au}$ $4 \mathrm{f}_{7 / 2}$ at $84 \mathrm{eV}$, and of $\mathrm{Ag} 3 \mathrm{~d}_{5 / 2}$ at $368.1 \mathrm{eV}$ ). Valence band spectra were aligned at the Fermi Edge. The fitting of all XPS was done using a Shirley background and Voigt integral functions, while the valence band spectra were deconvoluted using a Fermi step function and a set of Gaussian functions. Due to the high complexity of the C 1s and VB spectra of the bilayers, the Lorentzian width, FWMH, and intensity ratio among the different components of the same species are inferred from the analysis of the pure monolayer and multilayer spectra and kept almost fixed across the bilayer phase spectra, while BEs are left as free parameters of the fit (further details on the fitting parameteres are presented in the SI). STM images of monolayer and bilayers were measured at room temperature in a commercial Aarhus STM operated with Nanonis control electronics (both 
provided by SPECS) in constant current mode. The analysis of the STM images has been performed with the freeware WSxM from Nanotec ${ }^{19}$.

\section{Results and discussion}

\section{Second layer conformation}

Polarization-dependent NEXAFS can be employed to determine the adsorption geometry of aromatic molecules on surfaces ${ }^{20}$. To this aim, we performed NEXAFS measurements in two different configurations, i.e. with the electric field parallel (s-pol) and perpendicular (p-pol) to the surface. In Fig. 1, the $\mathrm{C}$ K-edge NEXAFS spectra for $\mathrm{F}_{16} \mathrm{CuPc} / \mathrm{PEN}: \mathrm{F}_{16} \mathrm{CuPc}$ (Fig. 1a) and PEN/PEN:F ${ }_{16} \mathrm{CuPc}($ Fig. 1b) bilayers on $\mathrm{Au}(111)$ are reported for p-pol and s-pol configurations (continuous and dotted lines, respectively). The ratio of the PEN:F ${ }_{16} \mathrm{CuPc}$ contact layer is $3: 1$. In Fig. 1c,d,e, the correspondent monolayer spectra of $\mathrm{F}_{16} \mathrm{CuPc}, \mathrm{PEN}$ and the 3:1 mixed phase are shown for comparison. The vanishing of $\pi^{*}$ resonances in s-pol spectra for all monolayer structures (Fig. 1c-e) indicates that, as long as they are in contact with the $\mathrm{Au}(111)$ surface, $\mathrm{F}_{16} \mathrm{CuPc}$ and PEN molecules adsorb flat. The face-on orientation of the contact layer has been previously observed by scanning tunneling microscopy ${ }^{16}$, and is generally ascribed to the strong interaction between metal $d$ bands and molecular $\pi$ states $^{21}$.

The absence of $\pi^{*}$ resonances in the s-pol spectra of the 3:1 contact layer makes it possible to properly evaluate the bilayers' structural conformation, i.e., whether the molecules of the second layer switch to a tilted configuration. This is indeed the case of PEN/3:1/Au(111) (Fig. 1b). The spol spectra shows a non-vanishing intensity in the low photon energy region corresponding to PEN LUMO and LUMO+1 (283-286 eV), which implies that pentacene molecules in the second layer are tilted with respect to the surface. From the angular dependence of LUMO components, a tilt angle of $30^{\circ} \pm 10^{\circ}$ with respect to the surface is deduced (see Supporting Info file for numerical details, Fig. S2). This angle is associated with a rotation of pentacene around its long axis, as 
determined in previous STM measurements ${ }^{16}$. The change from a face-on to such an edge-on configuration is not unexpected for PEN molecules beyond the molecule-surface contact ${ }^{22}$, and it is driven by the coupling of the $\mathrm{CH}$ edge bonds with the $\pi$-symmetry orbitals of the molecule underneath $^{23,24}$. In contrast, for $\mathrm{F}_{16} \mathrm{CuPc} / 3: 1 / \mathrm{Au}(111)$ we observe no intensity in the phthalocyanine $\pi$-region (284-286 eV) of the s-pol spectrum (Fig. 1 a), indicating that all the $\mathrm{F}_{16} \mathrm{CuPc}$ molecules remain flat in the second layer. These results are at odd with the interpretation of STM images in Ref. ${ }^{16}$, where a tilted configuration for $\mathrm{F}_{16} \mathrm{CuPc}$ molecules in the topmost layer was proposed. Note that in NEXAFS the molecular orientation is mirrored straightforwardly by the polarization dependence of the resonance intensity, whereas in STM the tunneling current is also a function of the local electronic density of states.

In the $\mathrm{F}_{16} \mathrm{CuPc} / 3: 1 / \mathrm{Au}(111)$ system the dichroism was also evaluated at the $\mathrm{N} \mathrm{K}$-edge spectra, which entirely belongs to absorption of $\mathrm{F}_{16} \mathrm{CuPc}$ molecules (inset of Fig. 1a). The p-pol spectrum is dominated by an intense peak at $398.3 \mathrm{eV}$, corresponding to transitions to the lowest unoccupied molecular orbital (LUMO), which disappears in the s-pol configuration. In the latter, the small peak at low photon energies corresponds to transitions to the $\mathrm{Cu} 3 \mathrm{~d}$-derived single unoccupied molecular orbital (SUMO), whose dipole moment lies in the plane of the molecule ${ }^{25,26}$. On $\operatorname{Ag}(111)$, a crystalline 3:1 contact-layer phase is not formed, being the 1:1 phase the stable one that allows for bilayer growth. The NEXAFS spectra for $\mathrm{F}_{16} \mathrm{CuPc} / \mathrm{PEN}: \mathrm{F}_{16} \mathrm{CuPc}$ and $\mathrm{CuPc} / \mathrm{CuPc}: \mathrm{PFP}$ bilayers grown on $\mathrm{Ag}(111)$ substrates are shown in Figs. 2a-d. As in $\mathrm{F}_{16} \mathrm{CuPc} / 3: 1 / \mathrm{Au}(111), \pi^{*}$-resonances are entirely quenched in both the $\mathrm{N}$ K-edge (Fig. 2a,b) and the C K-edge (Fig. 2c,d) for spolarization, demonstrating the face-on configuration for the phthalocyanine overlayer. The absence of intermixing, namely pentacene segregation at the molecular hetero-interface, has been checked by STM. The images show that a pure ordered layer of phthalocyanine is formed on top of the 1:1 buffer monolayer (Fig. S3).

\section{Molecular level realignment at bilayers}


The energy level alignment in multi-component molecular/metal systems can be transparently assessed through core-level photoemission when the molecular levels are essentially pinned to the vacuum level, as shown recently for donor:acceptor monolayers on noble metal surfaces ${ }^{13}$. Additionally, core-levels allow the clear identification of the whole variety of atomic species in the donor-acceptor complex. In Figs. 3a and 3b (middle spectra) we show C 1s photoemission spectra for $\mathrm{F}_{16} \mathrm{CuPc} / \mathrm{PEN}: \mathrm{F}_{16} \mathrm{CuPc}$ and $\mathrm{PEN} / \mathrm{PEN}: \mathrm{F}_{16} \mathrm{CuPc}$ bilayers on $\mathrm{Au}(111)$, respectively. In the same panels, the spectra of the contact mixed phase $\mathrm{PEN}: \mathrm{F}_{16} \mathrm{CuPc}$ (top) and the corresponding pure multilayer spectra (bottom) are also shown. Despite the apparent complexity, contributions from each molecular species, as well as from each of the layers, can be readily assigned. According to previous results $^{13,27,28}$, in the spectra of the contact blend shown on top of Figs. $3 \mathrm{a}$ and $3 \mathrm{~b}$, the three components at higher binding energy originate from the $\mathrm{F}_{16} \mathrm{CuPc}$ emission, and they derive from the different chemical environments of $\mathrm{C}$ atoms. The part of the spectrum at lower binding energy belongs to pentacene, and it can be satisfactorily reproduced by using two components. The present atomic decomposition straightforwardly applies by using a 3:1 intensity ratio between the two molecular species. Compared to the contact 3:1 spectrum, the bilayer structure shows extra emission at the high energy side of the $\mathrm{C} 1 \mathrm{~s}$ spectrum in $\mathrm{F}_{16} \mathrm{CuPc} / \mathrm{PEN}: \mathrm{F}_{16} \mathrm{CuPc}$, and at the low energy side in PEN/PEN: $\mathrm{F}_{16} \mathrm{CuPc}$ (middle spectra of Fig 3a and 3b, respectively). Such extra emission is assigned to the overlayer. It can be separated in the different atomic contributions, based on the respective multilayer spectra. Thus, we have well-separated contributions to the bilayer spectrum from the overlayer and the contact 3:1 layer.

Remarkably, upon capping, the core-levels of the contact layer "realign" with respect to the reference of the uncovered monolayer blend. In fact, after capping with $\mathrm{F}_{16} \mathrm{CuPc}$, the contact 3:1 emission shifts to lower binding energy. The same trend is observed in the PEN/PEN: $\mathrm{F}_{16} \mathrm{CuPc} / \mathrm{Au}(111)$, but in opposite directions, i.e. the 3:1 contact layer levels shift to higher binding energy upon PEN capping. The same core-level realignment trend observed in the 
contact layer of $\mathrm{F}_{16} \mathrm{CuPc} / \mathrm{PEN}: \mathrm{F}_{16} \mathrm{CuPc}$ on $\mathrm{Au}(111)$ occurs on $\mathrm{Ag}(111)$ (Fig. 3c). Note that all components belonging to the same layer shift almost rigidly. We also test the "swap" of the donoracceptor character, i.e., we consider the $\mathrm{CuPc} / \mathrm{CuPc}: \mathrm{PFP}$ bilayer. This bilayer system is morphologically analogous to the $\mathrm{F}_{16} \mathrm{CuPc} / \mathrm{PEN}: \mathrm{F}_{16} \mathrm{CuPc}$ bilayer, but with a donor-like capping (CuPc). In the $\mathrm{C} 1 \mathrm{~s}$ spectrum of the CuPc:PFP blend (Fig. 3d), the two components at lower binding energy belong to $\mathrm{CuPc}$, while the two components at higher binding energy origin from PFP. With the exception of the CuPc peaks in the contact layer, which remain at a fixed position, core-levels in CuPc/CuPc:PFP/Ag(111) realign as in the donor-capped PEN/PEN:F ${ }_{16} \mathrm{CuPc} / \mathrm{Au}(111)$ system (Fig. 3b). Thus, for the electron level alignment in bilayers what really matters is the donor/acceptor character of the top layer, and not the morphology of the molecular units.

Interestingly, also the core-levels of the capping layer "realign" with respect to the multilayer references. We can exclude the core level shift of the capping layer to be originated by a reduced core hole screening (i.e. a final state effect), as a consequence of the larger distance from the metallic surface, because i) the size of the core level shift in the capping layer is different from that of its multilayer reference and ii) the direction of the shift with respect to the multilayer depends on the donor/acceptor character of the capping layer. In fact, the $\mathrm{F}_{16} \mathrm{CuPc}$ capping layer peaks are shifted to higher binding energy compared to the pure $2 \mathrm{ML} \mathrm{F}_{16} \mathrm{CuPc}$ film reference shown on the bottom. For the bilayer blends covered by donor molecules, i.e. PEN/PEN:F ${ }_{16} \mathrm{CuPc} / \mathrm{Au}(111)$ (Fig. 3b) and $\mathrm{CuPc} / \mathrm{CuPc}: \mathrm{PFP} / \mathrm{Ag}(111)$ (Fig. 3d), the pure multilayer references correspond to $3 \mathrm{ML}$ of PEN/Au(111) and the 4 ML of CuPc/Ag(111) (bottom spectra of Fig. 3b and 3d, respectively). The overlayer peaks of donor molecules exhibit a shift to lower binding energy compared to the pure multilayer reference, i.e. in opposite direction with respect to what is observed for the acceptor overlayer.

In contrast to core-level spectra, donor and acceptor valence band (VB) levels overlap within a narrow energy range. However, the simultaneous analysis of core and frontier levels is needed to 
provide a clear understanding of the electronic structure in such entangled systems. The realignment trend observed for core-levels upon bilayer capping is also observed in molecular orbitals at the valence band (VB), demonstrating a quasi-rigid electronic structure for the bilayer system. In Fig. 4 we show VB spectra of bilayer systems featuring a second layer of phthalocyanine. As in C 1s, the $\mathrm{HOMO}$ resonances can be distinctly resolved for each molecule at each layer. $\mathrm{F}_{16} \mathrm{CuPc}$ and $\mathrm{CuPc}$ on $\operatorname{Ag}(111)$ also show the LUMO-derived feature (F-LUMO) at the Fermi Edge, revealing its partial filling through charge transfer from the substrate ${ }^{14}$. After capping with $\mathrm{F}_{16} \mathrm{CuPc}$ (acceptor, Fig. 4a and $4 \mathrm{~b}$ ), all the molecular levels in the contact layer shift downward in energy, in agreement with the $\mathrm{C}$ 1s spectra. The CuPc/CuPc:PFP/Ag(111) bilayer exhibits the reverse behavior, with the exception of a fixed position for the CuPc contact layer peaks (Fig. 4c). In accordance with the respective $\mathrm{C}$ 1s spectra (Fig. $3 \mathrm{c}$ ), the $\mathrm{HOMO}$ of the $\mathrm{F}_{16} \mathrm{CuPc}$ and $\mathrm{CuPc}$ overlayers appears shifted to higher and lower binding energy, respectively, compared to the thicker film reference.

The molecular-level realignment in donor/acceptor blends may be explained by the influence of the local electrostatic potential in molecular orbital energies. As discussed for monolayer blends of variable composition and stoichiometry ${ }^{13}$, the relative acceptor/donor character of the mixture affects the energy levels of the molecular complex as a whole. Stoichiometry-dependent energy level shifts could be explained in terms of the change in the average electrostatic potential probed by each of the molecules, which is more attractive for donor-like blends compared to acceptor-like mixtures ${ }^{29}$. Here we have shown that this scenario can be extended in the vertical direction, e.g., to bilayer blend structures. We first focus on the molecular levels of the blend contacting the metal. These are observed to shift almost rigidly (i.e. both donor and acceptor core levels) to lower or higher binding energy, depending on whether the capping layer has an acceptor or donor character. Analogously to the monolayer case, the observed realignment of core levels may be explained in terms of the changes in the average electrostatic potential caused by the capping overlayer. Specifically, a donor overlayer creates a more binding potential than an acceptor overlayer, shifting 
core levels of the contact layer blend to higher binding energy and viceversa. This is exemplified in Fig. 5 for the $\mathrm{F}_{16} \mathrm{CuPc}$ and PEN case on $\mathrm{Au}(111)$, where it can be seen how the shifts caused by the capping layer follow the same logic as those previously reported for varying donor to acceptor ratio in the contact layer ${ }^{13}$.

A detailed quantitative analysis of all core and valence level shifts upon capping shows minor deviations from the strictly rigid band structure scenario, which in turn reveals, as previously discussed for monolayer blends ${ }^{13}$, differences in the way each molecular level "screens" the capping process, i.e., the changes in the molecule environment. To appropriately evaluate deviations to the rigid electronic structure shift at each contact layer, in Fig. 6 we summarize the binding-energy shifts of all core and VB levels, molecule by molecule, and system by system. The sign and magnitude of the shift is the same in each pair of contact molecules, except for CuPc (see discussion below). The smallest realignment shift corresponds to the $\mathrm{C}-\mathrm{N}$ component (triangles) of phthalocyanines, while in acceptor molecules the largest shift occurs for the C-F core-level line (squares). Differences among core-levels are likely connected with the subtle variations of the atomic distance to the metal surface observed within the same molecule ${ }^{30}$, which in turn can lead to a variable ability to screen changes in the electrostatic potential. Indeed, $\mathrm{CuPc}, \mathrm{F}_{16} \mathrm{CuPc}$ and $\mathrm{PFP}$ molecules on metals adsorb in a lying-down but distorted configuration, with the central $\mathrm{C}$ and $\mathrm{N}$ atoms exhibiting the shortest molecule-substrate distance ${ }^{31,32}$, and the outer $\mathrm{C}$ and $\mathrm{F}$ atoms residing at the largest distance ${ }^{29,32,33}$. For pentacene molecules in $\mathrm{F}_{16} \mathrm{CuPc} / 3: 1 / \mathrm{Au}(111)$ (Fig. 6a) the HOMO level shifts by a larger amount relative to core-levels. In this case the different shift is connected with the capping-induced transfer of charge to the metal (see next paragraph). Such charge depletion may trigger both, a distinct "chemical" shift among valence and core-levels, and a different screening of the photohole (photoemission final state).

Next we consider the reverse process, namely the realignment of the electronic levels of the capping overlayer, induced by a change in the contact layer composition. We observe the same qualitative 
behavior: compared to pure thick-layer spectra, the overlayer peaks are shifted to higher (lower) binding energy if we change the contact layer composition from pure acceptor (donor) to a blend. Moreover, the same picture also applies to the three-layer structures shown in Fig. 7. In this case, we fix the chemical nature of the outermost layer (pure pentacene) and modify the acceptor/donor composition of the molecular layer underneath. Both core-levels and valence orbitals of the top capping pentacene reach the minimum binding energy for the maximum acceptor content of the molecular blend underneath, i.e. the bilayer $\mathrm{F}_{16} \mathrm{CuPc} / \mathrm{PEN}: \mathrm{F}_{16} \mathrm{CuPc}$ (see Supporting Info for details on the VB spectra. Fig. S4).

The present results are consistent with previous observations made for a SnPc layer capping the PTCDA/Ag(111) interface ${ }^{8}$. In fact, electronic levels in the donor-like SnPc overlayer were observed to shift to lower binding energies with respect to the SnPc film, as in the donor-capped CuPc/CuPc:PFP/Ag(111) and PEN/PEN:F ${ }_{16} \mathrm{CuPc} / \mathrm{Au}(111)$ bilayers reported here. The authors explain the shift as the result of the formation of an interface dipole at the heterorganic interface, which is stronger than the interface dipole created in the pure SnPc multilayer. Assuming such heterorganic interface dipole scenario, here we show that the sign of this dipole is reversed by swapping the relative donor/acceptor character of the capping overlayer. Disregarding the nature or location of the electrostatic potential that shifts rigidly molecular levels, there is a clear donor/acceptor-ratio dependence, which applies to all species/layers/structures analyzed here, except for $\mathrm{CuPc}$ molecules in the contact layer. For $\mathrm{CuPc}$ we observe energy level pinning, as for PTCDA in CuPc/PTCDA/Ag(111) ${ }^{10}$. Pinning can be explained as due to a high density of molecule/metal hybrid states at $\mathrm{E}_{\mathrm{F}}$, which can accommodate charge transfers without major energy shifts, and, what appears to be more important, screen the surrounding electrostatic potential effectively. In fact, a high density of (F-LUMO) states at $E_{F}$ is observed in both CuPc/CuPc:PFP/Ag(111) and $\mathrm{F}_{16} \mathrm{CuPc} / \mathrm{PEN}: \mathrm{F}_{16} \mathrm{CuPc} / \mathrm{Ag}(111)$, although electronic level pinning only occurs for $\mathrm{CuPc}$. This suggests that the hybrid, metallic character, and hence the ability of 
screening the electrostatic potential is stronger for F-LUMO states in $\mathrm{CuPc}$ as compared to $\mathrm{F}_{16} \mathrm{CuPc}$. The different degree of metallic hybridization goes along with the notably distinct molecule-metal distance for donor-like $\mathrm{CuPc}$ and acceptor-like $\mathrm{F}_{16} \mathrm{CuPc}$. For single-component monolayers on $\mathrm{Ag}(111)$, the reported value for $\mathrm{CuPc}$ is $2.97 \AA^{31}$, i.e., much smaller than the $3.25 \AA$ for $\mathrm{F}_{16} \mathrm{CuPc}^{32}$. In a contact blend layer, the adsorption height of $\mathrm{F}_{16} \mathrm{CuPc}$ is expected to increase further, in accordance to previous observations for acceptor-like molecules in donor:acceptor monolayer blends ${ }^{15,29}$.

\section{Charge transfer upon realignment}

As shown in molecular contact layers with variable composition, the changing of supramolecular environment may drive a rigid shift of the molecules' electronic states ${ }^{13}$. By tuning the electronic level alignment through these means, one can trigger charge transfer across the molecule/metal interfaces $^{14,15}$. In particular, a downward or upward energy level shift leads to charge transfer from or to the metal substrate, respectively. An excellent way to probe potential charge transfer upon energy level realignment is by the simultaneous analysis of the Fermi Edge $\left(\mathrm{E}_{\mathrm{F}}\right)$ intensity in valence-band photoemission spectra and NEXAFS ${ }^{14,34,35}$.

In all cases analyzed here the capping layer leads to the attenuation of the Fermi Edge intensity in the valence band spectra (Fig. 4). The attenuation reflects the fact that the topmost molecular layer does not add a significant density of $E_{F}$ states to the system, i.e.it has pure semiconducting character. On the other hand, it makes it difficult to assess spectral intensity changes in the contact layer $^{11} . E_{F}$ changes in this case are better studied through atom-selective NEXAFS. In Fig. 8 we consider $\mathrm{F}_{16} \mathrm{CuPc} / \mathrm{PEN}: \mathrm{F}_{16} \mathrm{CuPc}$ bilayers on $\mathrm{Au}(111)$ and $\mathrm{Ag}(111)$. Both keep the planar adsorption geometry in the second layer, such that spectral density variations can be directly linked to changes in the density of states. 
First we focus on pentacene, which is only present at the contact layer, by looking at the lowest energy side (283-284 eV) of the NEXAFS C-K edge spectra (Fig. 8). This range belongs to the pentacene LUMO region, featured with three different peaks, which are well-separated from the $\mathrm{F}_{16} \mathrm{CuPc}$ LUMO contribution at higher energy (dotted line). However, this part of the spectrum also contains the pentacene Fermi Edge at $283.55 \mathrm{eV}$, namely the energy onset for transitions from the pentacene core-level into any eventual pentacene's metallized state. The appearance of the pentacene's LUMO at the Fermi energy is due to the core-hole exciton shift ${ }^{20}$. On $\operatorname{Ag}(111)$, the pentacene's LUMO features remain completely unchanged upon capping (Fig. 8a). In contrast, the capping of the contact layer on $\mathrm{Au}(111)$ leads to extra absorption intensity right at the Fermi Edge, between the first two LUMO features (shaded area of Fig. 8b). This effect relates to excitations into considerably metallized molecular states and thus evidences noticeable pentacene/Au hybridization. In particular, the previously described energy level shift of the valence band upon bilayer growth causes the tail of the metallized and broadened HOMO (at binding energy of $0.62 \mathrm{eV}$ ) to become unoccupied. The intensity changes in NEXAFS are thus understood as a crossing above $\mathrm{E}_{\mathrm{F}}$ of the sizeable tail of metallized HOMO state (see valence band in Fig. 4a), which in turn occurs as the energy levels realign upon overlayer adsorption. This is in line with the energy level alignment of pentacene on $\mathrm{Au}(111)$, which shows the HOMO level to lie closer to Fermi than the $\mathrm{LUMO}^{36}$. In the case of $\operatorname{Ag}(111)$, the same generalized shift of the valence band is not affecting the pentacene NEXAFS because the corresponding pentacene HOMO lies at much higher binding energy of $\sim 1.6$ $\mathrm{eV}$ (see valence band in Fig. 4b). Note also that in the case of $\mathrm{Au}(111)$ the now partially unoccupied HOMO and exciton-shifted LUMO resonance happen to coincide. This is explained by considering that core-hole exciton effects are large for pure molecular levels peaks, such as pentacene's LUMO, but negligible for metallic states, leading to an apparent overlap of both structures at the same energy in absorption spectra ${ }^{20}$. 
A similar scenario is expected for the $\mathrm{F}_{16} \mathrm{CuPc}$ molecule in the contact layer upon growth of the capping layer. The collection of NEXAFS spectra at the $\mathrm{N} \mathrm{K}$-edge allows us to single out the unoccupied levels of $\mathrm{F}_{16} \mathrm{CuPc}$ from the pentacene ones, however a quantitative analysis of the NEXAFS spectral density in the contact layer is practically prevented by the overlap to the largely dominant contribution of the $\mathrm{F}_{16} \mathrm{CuPc}$ capping layer (see Supporting Information, Fig. S5).

\section{Conclusions}

Combining high resolution $\mathrm{X}$-ray photoemission, valence band photoemission and Near-Edge $\mathrm{X}$ ray absorption we have examined electronic states in bilayer/metal interfaces, made of different donor/acceptor blends. We restrict ourselves to systems that exhibit sharp hetero-molecular interfaces, without interlayer mixing. This requires metal-contact layer blends, either the 3:1 PEN: $F_{16} \mathrm{CuPc}$ combination on $\mathrm{Au}(111)$, or the 1:1 PEN:F ${ }_{16} \mathrm{CuPc}$ and 1:1 CuPc:PFP mixtures on $\operatorname{Ag}(111)$. Such contact layers exhibit quasi-rigid electronic structure shifts depending on whether they are capped by donor-like $(\mathrm{PEN}, \mathrm{CuPc})$ or acceptor-like $\left(\mathrm{F}_{16} \mathrm{CuPc}\right)$ molecules. Notably, the overlayer electronic structure also shifts with respect to thick films following the same criterion, namely the relative donor/acceptor character of the contact layer. Shifts in electronic levels are important, since they trigger charge transfer across the interface affecting the contact layer, as proved with NEXAFS. Such quasi-rigid, donor/acceptor shifts are explained assuming a variable, local electrostatic potential that molecules sense upon mixing or capping with other species. For a particular case, namely CuPc contacting the metal, such electrostatic potential is fully screened, and electronic levels appear pinned.

\section{Acknowlegnment}

This work was supported by the Spanish Grant Nos. MAT2010-21156-C03-01, PIB2010US-00652, and the Basque Government Grant No. IT-621-13. We acknowledge funding from the European 
Community's Seventh Framework Programme (FP7/2007-2013) under Grant No. 226716 and under grant agreement $n^{\circ} 312284$.

SUPPORTING INFORMATION. Deconvolution of the C 1s spectrum showing the Shirley background (Fig. S1). Deconvolution of NEXAFS C K-edge spectra of PEN/PEN: $\mathrm{F}_{16} \mathrm{CuPc}$ on $\mathrm{Au}(111)$ taken under p-polarization and s-polarization (Fig. S2). STM images in constant current mode of $\mathrm{F}_{16} \mathrm{CuPc} / \mathrm{PEN}: \mathrm{F}_{16} \mathrm{CuPc} / \mathrm{Ag}(111)$ and $\mathrm{CuPc} / \mathrm{PFP}: \mathrm{CuPc} / \mathrm{Ag}(111)$ (Fig. S3). Valence band and NEXAFS C K-edge spectra of the PEN/F ${ }_{16} \mathrm{CuPc} / 3: 1$ three-layer on $\mathrm{Au}(111)$ (Fig. S4). NEXAFS N K-edge spectra of $\mathrm{F}_{16} \mathrm{CuPc} / \mathrm{PEN}: \mathrm{F}_{16} \mathrm{CuPc}$ on $\mathrm{Au}$ (111) and on $\operatorname{Ag}(111)$ (Fig. S5). This material is available free of charge via the Internet at http://pubs.acs.org.

\section{REFERENCES}

${ }^{1}$ Ishii, H.; Sugiyama, K.; Ito, E.; Seki, K. Energy Level Alignment and Interfacial Electronic Structures at Organic/Metal and Organic/Organic Interfaces. Adv. Mater. 1999, 11, 605-625.

${ }^{2}$ Hwang, J.; Wan, A.; Kahn, A. Energetics of metal-organic interfaces: New experiments and assessment of the field. Materials Science and Engineering 2009, 64, 1-31.

${ }^{3}$ Goiri, E.; Borghetti, P.; El-Sayed, A.; Ortega, J.E.; de Oteyza, D. G. Multi-component Organic Layers on Metal Substrates. Adv. Mater. doi:10.1002/adma.201503570.

${ }^{4}$ Flores, F.; Ortega, J.; Vazquez, H. Modelling Energy Level Alignment at Organic Interfaces and Density Functional Theory. Phys. Chem. Chem. Phys. 2009, 11, 8658-8675.

${ }^{5}$ Heimel, G.; Duhm, S.; Salzmann, I.; Gerlach, A.; Strozecka, A.; Niederhausen, J.; Burker, C.; Hosokai, T.; Fernandez-Torrente, I.; Schulze, G.; et. al. Charged and metallic molecular monolayers through surface-induced aromatic stabilization. Nat. Chem. 2013, 5, 187-194. 
6 Kleimann, C.; Stadtmüller, B.; Schröder, S.; Kumpf, C. Electrostatic Interaction and Commensurate Registry at the Heteromolecular $\mathrm{F}_{16} \mathrm{CuPc}-\mathrm{CuPc}$ Interface. J. Phys. Chem. C 2014, $118,1652-1660$.

${ }^{7}$ Chen, W.; Huang, H.; Chen, S.; Gao, X. Y.; Wee, A. T. S. Low-Temperature Scanning Tunneling Microscopy and Near-Edge X-ray Absorption Fine Structure Investigations of Molecular Orientation of Copper(II) Phthalocyanine Thin Films at Organic Heterojunction Interfaces. J. Phys. Chem. C 2008, 112, 5036-5042.

${ }^{8}$ Häming, M.; Greif, M.; Sauer, C.; Schöll, A.; Reinert, F. Electronic structure of ultrathin heteromolecular organic-metal interfaces: SnPc/PTCDA/Ag(111) and SnPc/Ag(111). Phys. Rev. B 2010, 82, 235432.

${ }^{9}$ Gruenewald, M.; Sauer, C.; Peuker, J.; Meissner, M.; Sojka, F.; Scholl, A.; Reinert, F.; Forker, R.; Fritz, T.; Commensurism at electronically weakly interacting phthalocyanine/PTCDA heterointerfaces. Phys. Rev. B 2015, 91, 155432.

${ }^{10}$ Stadtmüller, B.; Sueyoshi, T.; Kichin, G.; Kroger, I.; Soubatch, S.; Temirov, R.; Tautz, F. S.; Kumpf, C. Commensurate Registry and Chemisorption at a Hetero-organic Interface. Phys. Rev. Lett. 2012, 108, 106103.

${ }^{11}$ Stadtmüller, B.; Willenbockel, M.; Schroder, S.; Kleimann, C.; Reinisch, E. M.; Ules, T.; Soubatch, S.; Ramsey, M. G.; Tautz, F. S.; Kumpf, C. Modification of the PTCDA-Ag bond by forming a heteromolecular bilayer film. Phys. Rev. B 2015, 91, 155433.

12 Amsalem, P.; Niederhausen, J.; Frisch, J.; Wilke, A.; Broker, B.; Vollmer, A.; Rieger, R.; Mullen, K.; Rabe, J. P. ; Koch, N.; Metal-to-Acceptor Charge Transfer through a Molecular Spacer Layer. J. Phys. Chem. C 2011, 115, 17503-17507.

${ }^{13}$ El-Sayed, A.; Borghetti, P.; Goiri, E.; Rogero, C.; Floreano, L.; Lovat, G.; Mowbray, D. J.; Cabellos, J. L.; Wakayama, Y.; Rubio, A. et al. Understanding Energy-Level Alignment in DonorAcceptor/Metal Interfaces from Core-Level Shifts. ACS Nano 2013, 7, 6914-6920. 
${ }^{14}$ Borghetti, P.; El-Sayed, A.; Goiri, E.; Rogero, C.; Lobo-Checa, J.; Floreano, L.; Ortega, J. E.; de Oteyza, D. G. Spectroscopic Fingerprints of Work-Function-Controlled Phthalocyanine Charging on Metal Surfaces. ACS Nano 2014, 12, 12786-12795.

15 Stadtmüller, B.; Lüftner, D.; Willenbockel, M.; Reinisch, E. M.; Sueyoshi, T.; Koller, G.; Soubatch, S.; Ramsey, M. G.; Puschnig, P.; Tautz, F. S.; et. al. Unexpected interplay of bonding height and energy level alignment at heteromolecular hybrid interfaces. Nat. Comm., 2014, 5, 3685 .

${ }^{16}$ Wakayama, Y.; de Oteyza, D. G.; Garcia-Lastra, J. M.; D. J. Mowbray. Solid-State Reactions in Binary Molecular Assemblies of $\mathrm{F}_{16} \mathrm{CuPc}$ and Pentacene. ACS Nano 2011, 5, 581-589.

${ }^{17}$ Bavdek, G.; Cossaro, A.; Cvetko, D.; Africh, C.; Blasetti, C.; Esch, F.; Morgante, A.; Floreano, L. Pentacene Nanorails on Au(110). Langmuir 2008, 24, 767-772.

${ }^{18}$ Floreano, L.; Cossaro, A.; Gotter, R.; Verdini, A.; Bavdek, G.; Evangelista, F.; Ruocco, A.; Morgante, A.; Cvetko, D. Periodic Arrays of Cu-Phthalocyanine Chains on $\mathrm{Au}(110)$. J. Phys. Chem. C 2008,112, 10794-10802.

${ }^{19}$ Horcas, I.; Fernandez, R.; Gomez-Rodriguez, J. M.; Colchero, J.; Gomez-Herrero, J.; Baro, A. M. WSXM: A software for scanning probe microscopy and a tool for nanotechnology. Rev. Sci. Instrum. 2007, 78, 013705-(1-8).

${ }^{20}$ Stohr, J. NEXAFS Spectroscopy 1992, Springer-Verlag Berlin Heidelberg.

21 Chen, W.; Huang, C.; Gao, X. Y.; Wang, L.; Zhen, C. G.; Qi, D. C.; Chen, S.; Zhang, H. L.; Loh, K. P.; Chen, Z. K.; et. al. Tuning the Hole Injection Barrier at the Organic/Metal Interface with Self-Assembled Functionalized Aromatic Thiols. J. Phys. Chem. B 2006, 110, 26075-26080.

${ }^{22}$ S. Sönchen, S. Lukas, G. Witte, Epitaxial growth of pentacene films on $\mathrm{Cu}(110)$. J. Chem. Phys. 2004, 121, 525-534.

${ }^{23}$ Lanzilotto, V.; Sanchez-Sanchez, C.; Bavdek, G.; Cvetko, D.; Lopez, M. F.; Martin-Gago, J. A.; Floreano, L. Planar Growth of Pentacene on the Dielectric TiO2(110) Surface. J. Phys. Chem. C 2011, 115, 4664-4672. 
${ }^{24}$ Martinez, C.R.; Iverson, B. L. Rethinking the term “pi-stacking”. Chem. Sci. 2012, 3, 2191-2201.

${ }^{25}$ de Oteyza, D. G.; Sakko, A.; El-Sayed, A.; Goiri, E.; Floreano, L.; Cossaro, A.; Garcia-Lastra, J. M.; Rubio, A.; Ortega, J. E. Inversed Linear Dichroism in F K-Edge NEXAFS Spectra of Fluorinated Planar Aromatic Molecules. Phys. Rev. B 2012, 86, 075469.

26 Nardi, M. V.; Detto, F.; Aversa, L.; Verucchi, R.; Salviati, G.; Iannotta, S.; Casarin, M. Electronic Properties of CuPc and H2Pc: An Experimental and Theoretical Study. Phys. Chem. Chem. Phys. 2013, 15, 12864-12881.

${ }^{27}$ El-Sayed, A.; Mowbray, D. J.; Garcia-Lastra, J. M.; Rogero, C.; Goiri, E.; Borghetti, P.; Turak, A.; Doyle, B. P.; Dell'Angela, M.; Floreano,L.; et al.Supramolecular Environment-Dependent Electronic Properties of MetalOrganic Interfaces. J. Phys. Chem. C 2012, 116, 4780-4785.

${ }^{28}$ Piper, L. F. J.; Cho, S. W.; Zhang, Y.; DeMasi, A.; Smith, K. E.; Matsuura, A. Y.; McGuinness, C. Soft X-ray Spectroscopy Study of the Element and Orbital Contributions to the Electronic Structure of Copper Hexadecafluoro-Phthalocyanine. Phys. Rev. B 2010, 81, 045201.

${ }^{29}$ Cabellos, J.L.; Mowbray, D.J.; Goiri, E.; El-Sayed, A.; Floreano, L.; de Oteyza, D. G.; Rogero, C.; Ortega, J. E.; Rubio, A. Understanding Charge Transfer in DonorAcceptor/Metal Systems: A Combined Theoretical and Experimental Study. J. Phys. Chem. C 2012, 116, 17991-18001.

${ }^{30}$ Goiri, E.; Matena, M.; El-Sayed, A.; Lobo-Checa, J.; Borghetti, P.; Rogero, C.; Detlefs, B.; Duvernay, J.; Ortega, J. E.; de Oteyza, D. G. Self-Assembly of Bicomponent Molecular Monolayers: Adsorption Height Changes and Their Consequences. Phys. Rev. Lett. 2014, 112, 117602.

${ }^{31}$ Kroger, I.; Stadtmuller, B.; Stadler, C.; Ziroff, J.; Kochler, M.; Stahl, A.; Pollinger, F.; Lee, T.-L.; Zegenhagen, J.; Reinert, F.; et al. Submonolayer Growth of Copper-Phthalocyanine on $\operatorname{Ag}(111)$. New J. Phys. 2010, 12, 083038.

${ }^{32}$ Gerlach, A.; Schreiber, F.; Sellner, S.; Dosch, H.; Vartanyants, I. A.; Cowie, B. C. C.; Lee, T.-L.; Zegenhagen, J. Adsorption. Induced Distortion of F16CuPc on $\mathrm{Cu}(111)$ and $\mathrm{Ag}(111)$ : An X-ray Standing Wave Study. Phys. Rev. B 2005, 71, 205425. 
${ }^{33}$ Koch, N.; Gerlach, A.; Duhm, S.; Glowatzki, H.; Heimel, G.; Vollmer, A.; Sakamoto, Y.; Suzuki, T.; Zegenhagen, J.; Rabe, J. P.; et al. Adsorption-Induced Intramolecular Dipole: Correlating Molecular Conformation and Interface Electronic Structure. J. Am. Chem. Soc. 2008, 130, 73007304.

${ }^{34}$ Schöll, A.; Kilian, L.; Zou, Y.; Ziroff, J.; Hame, S.; Reinert, F.; Umbach, E.; Fink, R. H. Disordering of an Organic Overlayer on a Metal Surface Upon Cooling. Science 2010, 329, 303305

35 Jia, Z; Lee, V. W.; Kymissis, I.; Floreano, L.; Verdini, A.; Cossaro, A.; Morgante, A. In situ study of pentacene interaction with archetypal hybrid contacts: Fluorinated versus alkane thiols on gold Phys Rev. B 2010, 82, 125457.

${ }^{36}$ Soe, W.-H.; Manzano, C.; De Sarkar, A.; Chandrasekhar, N.; Joachim, C. Direct Observation of Molecular Orbitals of Pentacene Physisorbed on $\mathrm{Au}(111)$ by Scanning Tunneling Microscope. Phys. Rev. Letter 2009, 102, 176102. 


\section{Figures}

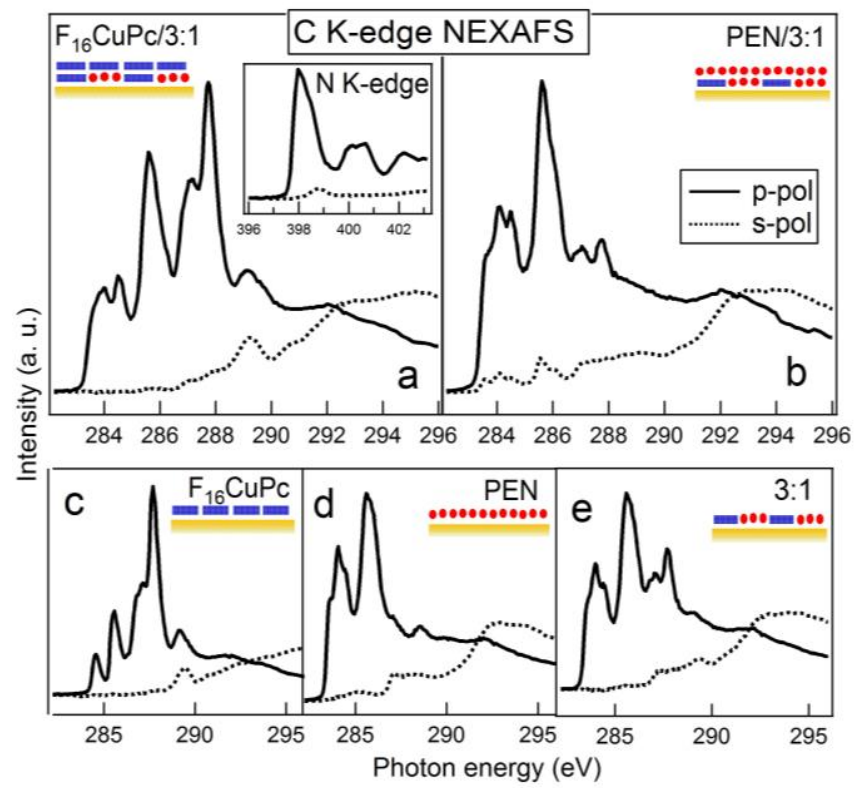

Figure 1. NEXAFS C K-edge spectra of (a) $\mathrm{F}_{16} \mathrm{CuPc} / \mathrm{PEN}$ : $\mathrm{F}_{16} \mathrm{CuPc}$, (b) PEN/PEN: $\mathrm{F}_{16} \mathrm{CuPc}$, (c) $1 \mathrm{ML}$ of $\mathrm{F}_{16} \mathrm{CuPc}$, (d) $1 \mathrm{ML}$ of PEN and (e) and PEN:F $\mathrm{F}_{16} \mathrm{CuPc}$ deposited on $\mathrm{Au}(111)$, where the PEN:F $\mathrm{F}_{16} \mathrm{CuPc}$ blend correspond to a 3:1 stoichiometry. In the inset of panel (a), NEXAFS N K-edge spectra of $\mathrm{F}_{16} \mathrm{CuPc} / 3: 1 / \mathrm{Au}(111)$ are also presented. Spectra are taken under p-polarization (solid lines) and s-polarization (dashed lines). The $\pi^{*}$ resonances ( $\left.\mathrm{h} v=283-286 \mathrm{eV}\right)$ are quenched in all s-pol spectra except for the PEN/PEN: $\mathrm{F}_{16} \mathrm{CuPc}$ bilayer (b), indicating a tilted configuration for pentacene molecules of the capping layer.

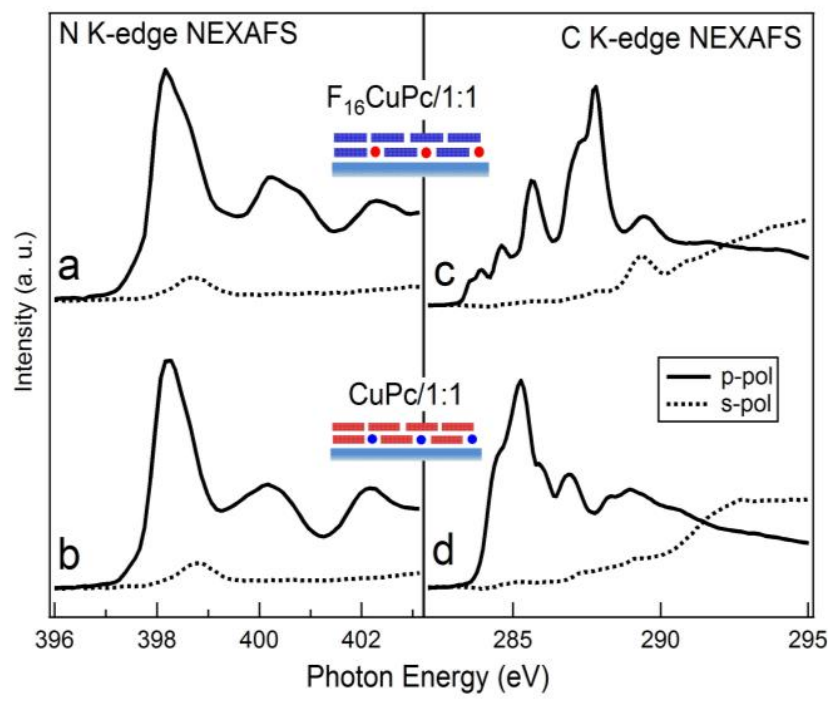

Figure 2. Left panel: NEXAFS N K-edge spectra of (a) $\mathrm{F}_{16} \mathrm{CuPc} / 1: 1 / \mathrm{Ag}(111)$ and (b) $\mathrm{CuPc} / 1: 1 / \mathrm{Ag}(111)$. Right panel: NEXAFS C K-edge spectra of (c) $\mathrm{F}_{16} \mathrm{CuPc} / 1: 1 / \mathrm{Ag}(111)$ and (d) $\mathrm{CuPc} / 1: 1 / \mathrm{Ag}(111)$. Spectra are taken under p- 
polarization (solid lines) and s-polarization (dashed lines). The absence of any $\pi^{*}$ resonances in the s-pol spectra indicates that both $\mathrm{F}_{16} \mathrm{CuPc}$ and $\mathrm{CuPc}$ are lying flat in the capping layer.

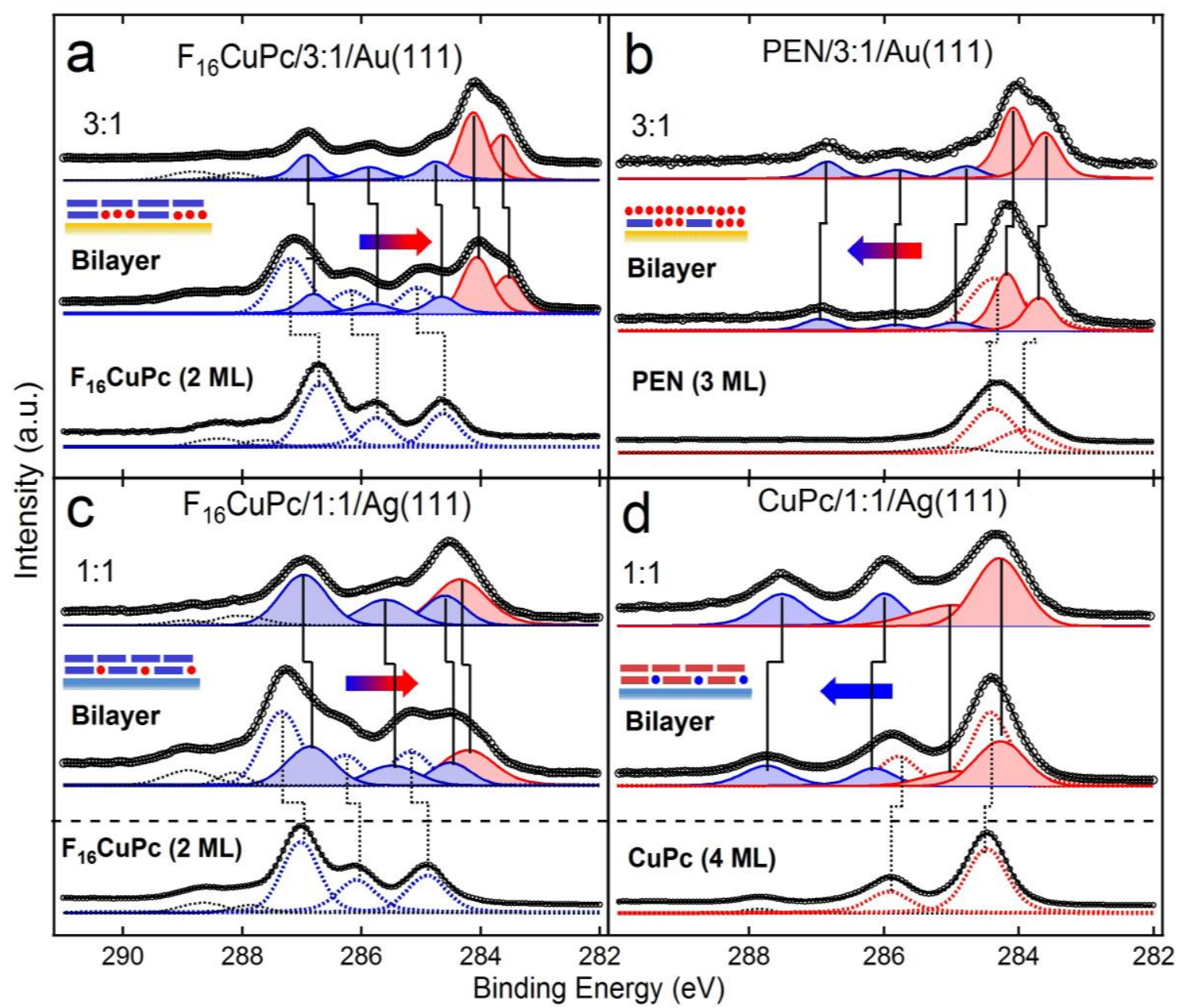

Figure 3. $\mathrm{C}$ 1s core levels of (a) $\mathrm{F}_{16} \mathrm{CuPc} / 3: 1 / \mathrm{Au}(111)$, (b) $\mathrm{PEN} / 3: 1 / \mathrm{Au}(111)$, (c) $\mathrm{F}_{16} \mathrm{CuPc} / 1: 1 / \mathrm{Ag}(111), \quad(\mathrm{d})$ $\mathrm{CuPc} / 1: 1 / \operatorname{Ag}(111)$. The spectra of the bilayers (middle part) are compared to the respective monolayer blends (top part) and to (a) $2 \mathrm{ML}$ of $\mathrm{F}_{16} \mathrm{CuPc} / \mathrm{Au}(111)$, (b) $3 \mathrm{ML}$ of PEN/Au(111), (c) $2 \mathrm{ML}$ of $\mathrm{F}_{16} \mathrm{CuPc} / \mathrm{Ag}(111)$, (d) $4 \mathrm{ML}$ of $\mathrm{CuPc} / \mathrm{Ag}(111)$ (bottom part). Experimental points (markers) are superimposed to fit spectra (solid line). The fit components belonging to the contact and capping layers are marked by shaded and empty areas, respectively, while the red and blue colors indicates the donor and acceptor character of the molecules, respectively. Core-levels of the contact and capping layers are observed to shift rigidly with respect to the monolayer and thin layer references, where the sign of the shift depends on whether the capping layer has a donor or acceptor character. 


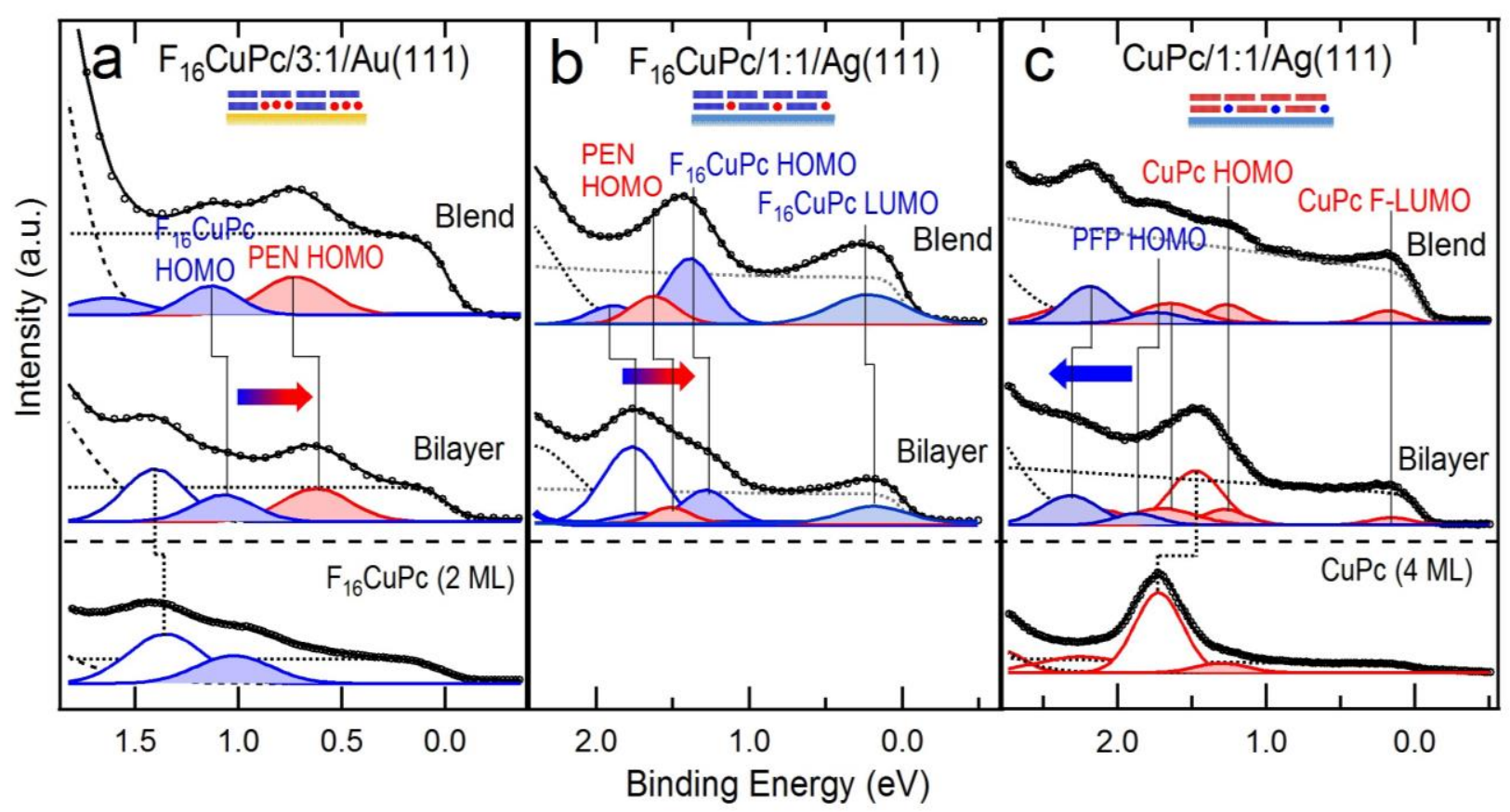

Figure 4. Valence band (VB) spectra of (a) $\mathrm{F}_{16} \mathrm{CuPc} / 3: 1 / \mathrm{Au}(111)$, (b) $\mathrm{F}_{16} \mathrm{CuPc} / 1: 1 / \mathrm{Ag}(111)$ and (c) $\mathrm{CuPc} / 1: 1 / \mathrm{Ag}(111)$. The spectra of the bilayers (middle part) are compared to the respective monolayer blends (top part) and to (a) 2 ML of $\mathrm{F}_{16} \mathrm{CuPc} / \mathrm{Au}(111)$ and (c) $4 \mathrm{ML}$ of $\mathrm{CuPc} / \mathrm{Ag}(111)$ (bottom part). The fit components belonging to the contact and capping layers are marked by shaded and empty areas, respectively, while the red and blue colors indicates the donor and acceptor character of the molecules, respectively. Valence band structures are observed to shifts in the same direction as the correspondent core-levels of Fig. 3.

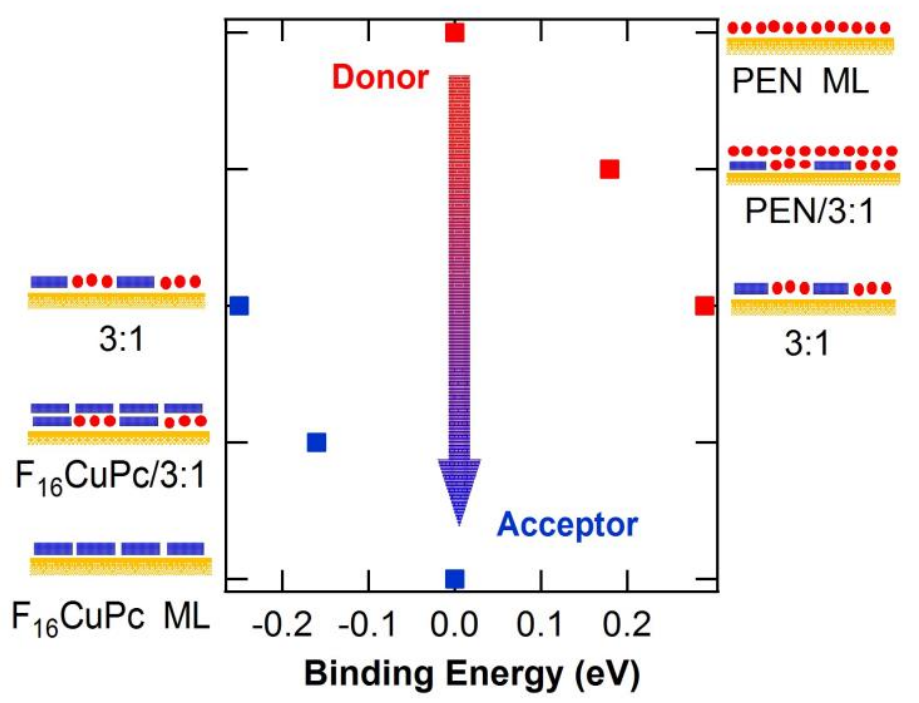

Figure 5. C 1s core levels shifts of acceptor (blue squares) and donor (red squares) molecules in the contact layer on $\mathrm{Au}(111)$ as the donor/acceptor ratio of the total system is varied. The reference values for pure monolayers on Au(111) are taken from Ref. ${ }^{13}$. 


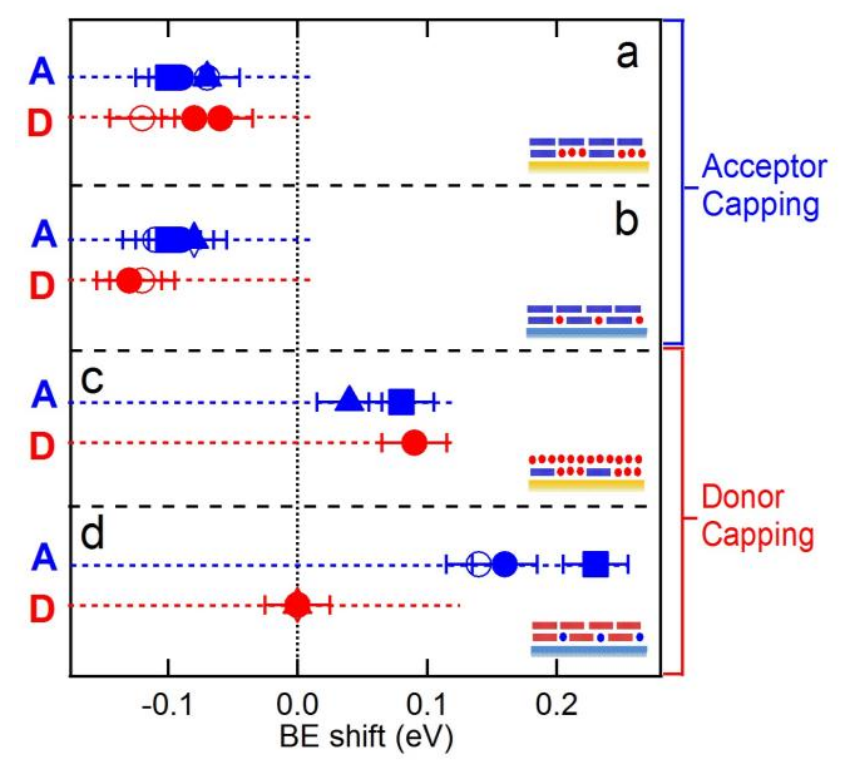

Figure 6. Realignment shifts of (a) $\mathrm{F}_{16} \mathrm{CuPc} / 3: 1 / \mathrm{Au}(111)$ (b) $\mathrm{F}_{16} \mathrm{CuPc} / 1: 1 / \mathrm{Ag}(111)$ (c) $\mathrm{PEN} / 3: 1 / \mathrm{Au}(111)$ and (d) $\mathrm{CuPc} / 1: 1 / \operatorname{Ag}(111)$. In each panel, the core-level and VB features shifts are shown for acceptors (top line A, blue markers) and donors (bottom line D, red markers) of the contact layer. Here, the core-level components of C-F, C-N, C$\mathrm{C}(\mathrm{C}-\mathrm{H})$ are marked by full squares, triangles and circles, respectively. The shift of the HOMO (circles) and F-LUMO (diamond-shape) features are represented by empty markers.

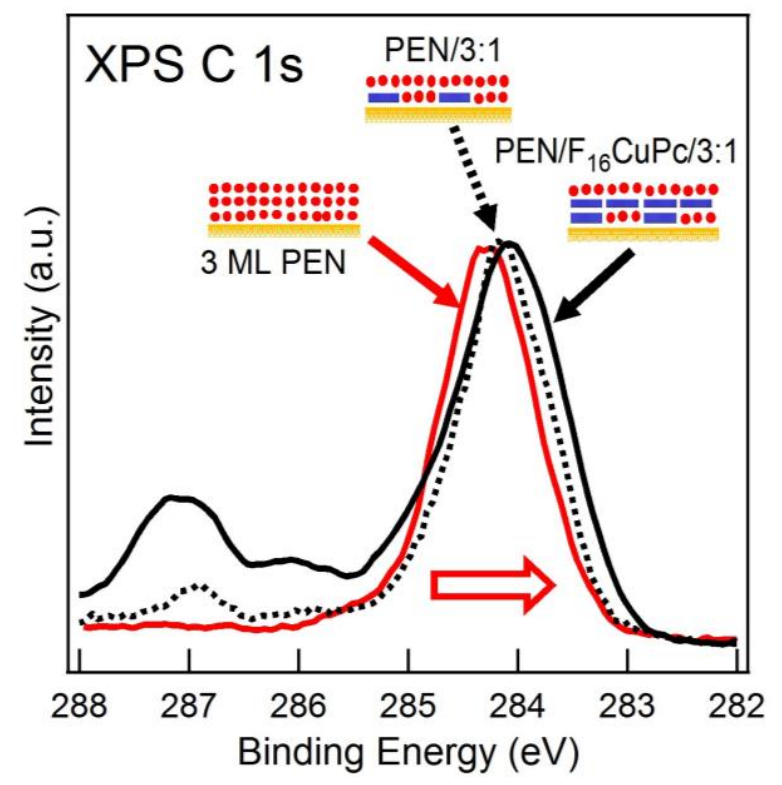

Figure 7. $\mathrm{C} 1$ s core levels of $3 \mathrm{ML}$ of PEN (red line), PEN/3:1 bilayer (black dotted line) and PEN/F $16 \mathrm{CuPc} / 3: 1$ threelayer (continuous line). While increasing the acceptor content of the molecular layers underneath (from left to right), the core-levels of pentacene $(\mathrm{BE}=283-285 \mathrm{eV})$ shift downward in energy. 


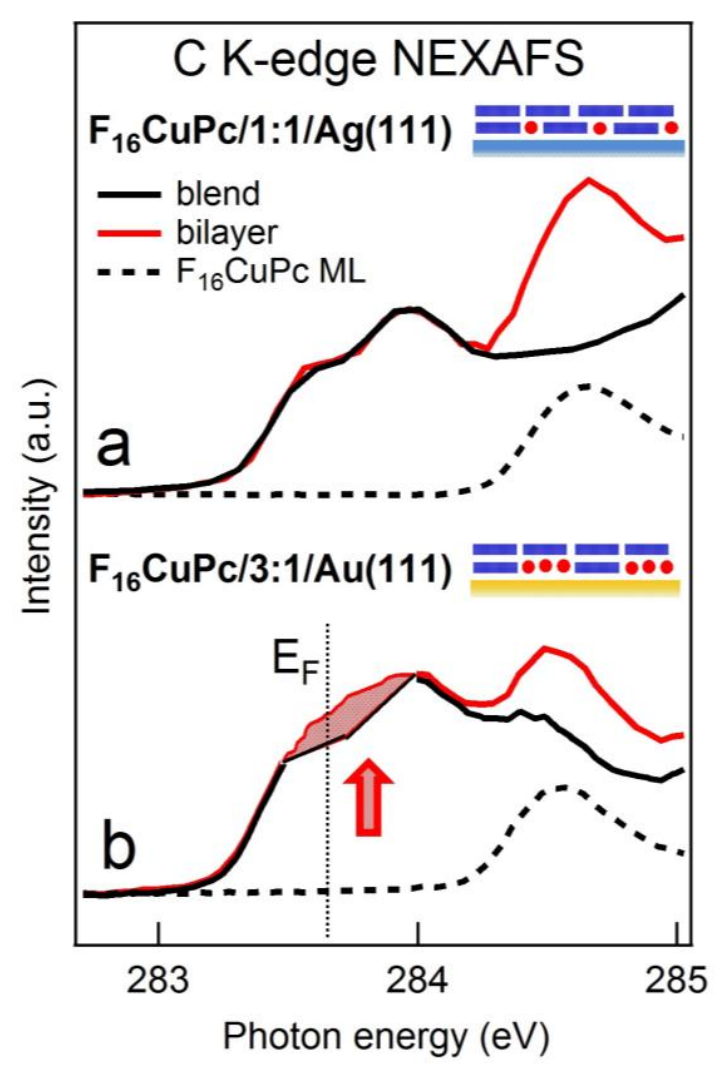

Figure 8. Left panels: NEXAFS C K-edge spectra of $\mathrm{F}_{16} \mathrm{CuPc} / \mathrm{PEN}: \mathrm{F}_{16} \mathrm{CuPc}$ (red curved) (a) on $\mathrm{Ag}$ (111) and (b) on $\mathrm{Au}(111)$ compared to their contact-layer references (black curves). The increase in intensity in the spectrum of bilayers on $\mathrm{Au}(111)$ compared to the contact-layer is marked by a shaded area. 
TABLE OF CONTENTS

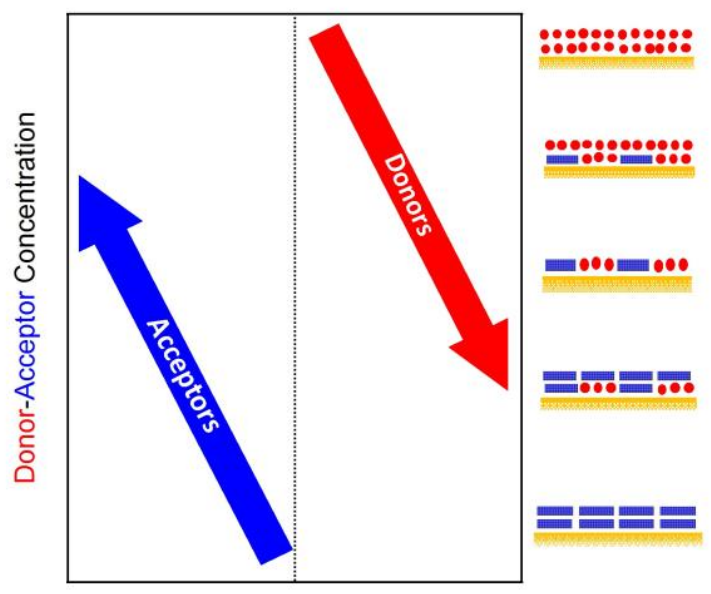

Binding Energy shift (eV) 